\title{
Synthesis and characterization of tin(IV) phenyl phosphonate in nano form
}

\author{
CHITRA SUMEJ* and BEENA RAVEENDRAN ${ }^{\dagger}$ \\ Department of Chemistry, S N College, Kollam 691 001, India \\ ${ }^{\dagger}$ Department of Chemistry, D B College, Kollam 691 021, India
}

MS received 7 November 2007; revised 23 February 2008

\begin{abstract}
An inorgano-organic ion exchanger, Sn(IV) phenyl phosphonate, has been synthesized in amorphous form. Further, an attempt has been made to synthesize Sn(IV) phenyl phosphonate in the nano form. The materials have been characterized for elemental analysis (ICP-AES), thermal analysis (TGA), X-ray analysis and FTIR spectroscopy. Chemical resistivity of these materials has been accessed in acidic, basic and organic solvent media. Catalytic activity has been studied and compared by using esterification of ethylene glycol as a model reaction wherein glycoldiacetate has been prepared. The transport properties of these materials have been explored by measuring specific proton conduction at different temperatures using SOLARTRON DATASET impedance analyser over a frequency range $1 \mathrm{~Hz}-1 \mathrm{MHz}$. It has been observed that Sn(IV) phenyl phosphate in the nano form behaves as a better Bronsted catalyst and proton conductor as compared to the amorphous form.
\end{abstract}

Keywords. Amorphous material; nano material; Sn(IV) phenyl phosphonate; protonic conductor.

\section{Introduction}

Nanotechnology is a term used to refer to material devices and structures measuring one billionth to 100 billionth of a meter. When we change the dimensions of matter in the order of a few nanometers, new properties are obtained. This will lead to the design of new ranges of functional materials and effects technological developments. The objective of nanotechnology is to create functional structures with dimensions in nanometer range. The size of nanoparticle is said to be $1 / 1000$ of the size of a hair.

The ultimate definition of nanotechnology is said to be the manipulation and control of individual atom and thereby the programmed formation of superstructures. Another definition is the organization of objects having nanodimensions such as molecules and other nanoscale matter such as quantum dots, bucky balls (also known as fullerenes) and nanotubes.

Organic molecules in the nanometer dimension are the basis of life. Nanophase materials can be organic or inorganic and essentially of any composition. The discovery of these materials by Gileiter in 1989 can be viewed as one of the most fascinating ones in recent times.

The nanocrystalline materials can be zero (clusters), one (lamellar), two (filamentary) or three (equiaxed particles) dimensional in nature and can be obtained by a number of techniques.

*Author for correspondence (chitrapg@hotmail.com)
Due to the high controlled size and structure selectivity of the nanomaterials, the physical and chemical properties of nanomaterials could be quite diverse. It is known that the properties of nanostructure depend strongly on their size and shape. An essential task in nanoscience is the property characterization of an individual nanostructure with well defined atomic structure.

Nanomaterials are classified into nanostructured materials and nanoparticle materials. The former refers to condensed bulk materials that are made up of grains with grain sizes in the nanometer size range, while the latter are usually the dispersive nanoparticles. Nanophase and nanostructured materials formed a new branch of materials research.

The exotic properties of nanoparticles have been considered in applications such as optoelectronics, catalysis, photochemical and non-linear optical devices. Quite recently, nano scale matter has been looked at with interest for potential applications in nanocomputers, synthesis of advanced materials for energy storage devices, chemical and biosensors as well as biomedical devices. They are also used as substrates for high temperature superconducting devices.

Application of small metal particles has attracted the attention of scientists for a long time. As early as in the seventies Turkevich and Kim (1970) already prepared monodispersed gold particles, and later using molecular carbonyl clusters (Guczi 1989), the importance of small nanoparticles increased considerably. Interest in nanomaterials is growing at a dramatic speed due to realiza- 
tion that induced dimensions in nanometer regime can alter the properties of matter (Ayyappan and Clearfield 2005).

Size reduction of metal particles leads to several changes in its surface properties. Small metal particles are more sensitive to the morphological environment, such as ion-metal interaction influencing also the electron properties of the metal surface. As small metal particles have a short range ordering, these particles are in metastable state, thus they tend to be stabilized by coalescence to form large metal particles.

Advantages of small metal particles are: (i) short range ordering, (ii) enhanced interaction with environments due to the high number of dangling bonds, (iii) great variety of the valence band electron structure and (iv) self-structuring for optimum performance in chemisorption and catalysis.

If the small metal particles are inserted into zeolite supercages, not only the size of the metals are confined according to the size of the supercage, but after reduction of the precursor metal ions in a stream of hydrogen, the protons replacing metal ions in the cation exchange positions also interfere with the metal particles influencing thereby their chemisorption and catalytic properties (Sakurai and Haruta 1996; Haruta 1997; Ribeiro et al 1997).

Inorgano-organic nanomaterials are a remarkable family of hybrid materials (Chitra and Beena 1997), which have been studied extensively because of their potential applicability to many industrial materials such as automobile, airplane, bridges, artificial tissues, contact lenses, bones, integrated circuits, insulators and others (Huang et al 1989). The thermal, electrical, optical, mechanical and biological properties are known to be widely controlled by adjusting compositions, nanophase size and the chemical bond between organic and inorganic phases (Honma et al 2001).

In the present study the nano form of an inorganoorganic ion exchanger, tin(IV) phenyl phosphonate (SnPPN), is prepared by the chemical co-precipitation method by using EDTA as a template. Nanoparticles of desired size and shape has enormous importance in nanotechnology (Gómez-Alcántara et al 2007). Depending on their size in nanoscale range, there occurs deviations of their physical-chemical properties (Haq et al 2004).

The synthesized material has been characterized for elemental analysis (ICP-AES), thermal analysis, FTIR, surface area measurements (BET) method, SEM, TEM, $\mathrm{X}$-ray diffraction studies and particle size determination. The catalytic activity of the material has been studied through the esterification of ethylene glycol and the proton conductivity is also measured by using an impedance analyser.

\section{Experimental}

\subsection{Synthesis of tin phenyl phosphonate (SnPPA)}

Tin phenyl phosphonate (SnPPA) was prepared by adding a solution of $\mathrm{Sn}(\mathrm{IV})$ chloride to an aqueous solution of phenyl phosphonic acid in $1: 1$ mole ratio with continuous stirring at $0 \mathrm{pH}$. The white precipitate obtained was kept overnight, filtered, washed with conductivity water till free of chloride ions and dried at $40^{\circ} \mathrm{C}$. The materials obtained were sized by sieving to 30-60 mesh size and converted to hydrogen form by immersion in $1 \mathrm{M} \mathrm{HCl}$.

Tin phenyl phosphonate-nano (SnPPN) was prepared by the chemical co-precipitation method by using EDTA as a template. The function of the template being a structure directing agent and regulating the pore size. Also the chelating agent, EDTA, kept the $\mathrm{Sn}^{4+}$ ions in homogeneous solution leaving enough flexibility for the system to exist homogeneously throughout the reaction and prepared more homogeneous nano sized materials of high purity.

\subsection{Chemical resistivity}

A study of chemical resistivity/stability of the synthesized material (SnPPN) in various chemical media is both useful and important while using the material for various applications in varied environments. $500 \mathrm{mg}$ each of the synthesized material was taken in $50 \mathrm{ml}$ of acids and bases of various concentrations as well as in organic solvents and allowed to stand for $24 \mathrm{~h}$ and the change in colour, nature and weight was noted.

\subsection{Characterization}

The sample was analysed for tin and phosphorus by ICPAES. Elemental analysis $(\mathrm{C}$ and $\mathrm{H})$ was done by using a Coleman analyser model 33.

Thermograms (TG) were recorded on a Perkin-Elmer thermal analyser at a heating rate of $10 \% \mathrm{~min}$. FTIR spectra were recorded on a Perkin-Elmer IR spectrophotometer. The particle size was determined from X-ray diffraction (using a Shimadzu XD 610 powder diffraction) data using Scherrer equation. SEM images of the samples were obtained using a scanning electron microscope. TEM image was obtained using PHILIPS CM 200 model. Specific surface area was calculated by using BET method. Adsorption desorption isotherms of nitrogen were recorded on a High speed Gas Sorption Analyser, Nova 1000, version $6 \cdot 07$

For the preparation of the ester, acetic acid and glycol was combined in a refluxing assembly, the nano catalyst was then added in the required amount. The mixture was heated to gentle reflux for $2 \mathrm{~h}$ and ester formed was distilled over slowly. Reactions were carried out by varying the amount of catalyst and mole ratio of alcohol and acid.

Proton conductivity of the material was measured in the temperature range $40-180^{\circ} \mathrm{C}$, at $20^{\circ} \mathrm{C}$ intervals, using a Solartron Dataset impedance analyser (S1 1260) over a frequency range $1 \mathrm{~Hz}-1 \mathrm{MHz}$. 


\section{Results and discussion}

Unlike the amorphous variety (SnPP) which was obtained as white granules, the nano form of tin phenyl phosphonate was obtained as a white fine powder. Elemental analysis indicates the following composition $(\mathrm{Sn}=20.659 \%, \mathrm{P}=$ $2.681 \%, \mathrm{C}=11.93 \%$ and $\mathrm{H}=2.66 \%$ ). Chemical resistivity $/$ stability of the material assessed in various chemical environments, shows that this material is stable in acidic (conc. $\mathrm{HCl}$ and $\mathrm{HNO}_{3}$ ), basic $(\mathrm{NaOH}$ and $\mathrm{KOH}$ ) and organic media (ethanol, glycol, benzene and acetic acid).

Thermogram of the material shows $20 \%$ weight loss at $200^{\circ} \mathrm{C}$, corresponding to the loss of external water molecules. There is a very gradual weight loss within the temperature range of $300-700^{\circ} \mathrm{C}$ corresponding to the decomposition of the organic moiety and the condensation of structural hydroxyl groups.

FTIR spectrum exhibits bands in the regions, $\sim 3400 \mathrm{~cm}^{-1}$ and $\sim 1630.02 \mathrm{~cm}^{-1}$, attributed to asymmetric and symmetric hydroxo-OH and aquo-OH stretches and of aquo $(\mathrm{H}-\mathrm{O}-\mathrm{H})$ bending, respectively. In addition to this, the FTIR spectrum also shows additional bands characteristic of the organic moiety. Band at $740.80 \mathrm{~cm}^{-1}$, being the out of plane $\mathrm{C}-\mathrm{H}$ ring bending mode and the bands at $1593.11,1485.17$ and $1438.87 \mathrm{~cm}^{-1}$ are due to $\mathrm{C}=\mathrm{C}$ stretching. Inplane bending bands occur in the region $1000-1200 \mathrm{~cm}^{-1}$.

Figure 1a exhibits the X-ray diffractogram of SnPPN and the diffractogram of SnPP (figure 1b) is also given for comparison. The X-ray diffractogram of SnPP does not show any peak, indicating an amorphous nature while SnPPN shows sharp well defined peaks. The average particle size from the diffraction pattern determined by using Scherrer equation is found to be $47 \mathrm{~nm}$ indicating the nano dimension of the material.

Figures $2 \mathrm{a}$ and $\mathrm{b}$ representing SEM images of SnPPN and SnPPA, respectively indicate irregular particle size. This is also supported by the particle size histogram of the samples. The specific surface areas (BET method) of SnPP and SnPPN were found to be 51.4755 and $113.2785 \mathrm{~m}^{2} / \mathrm{g}$, respectively.

Figures 3 and 4 representing TEM image and the arrhenius plot of SnPPN, indicate that these are formed by the aggregation of nanoparticles.

In order to study the catalytic property of the nanomaterial, esterification of ethylene glycol with acetic acid was selected as the model reaction.

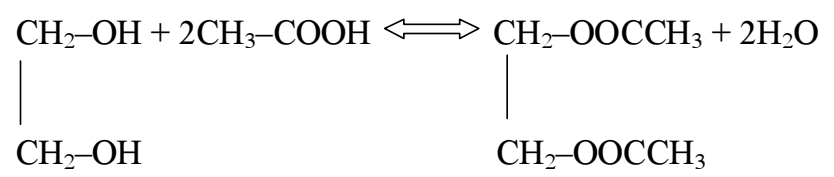

It was found that the yield of glycol diacetate increased by increasing the amount of catalyst and acid concentration. However, maximum yield was obtained when the amount of the catalyst taken was $2 \mathrm{~g}$ and glycol to acid proportion was $1: 2$. The yield of glycol diacetate was higher for SnPPN than SnPP used under similar conditions. The maximum yield for SnPPN was $75 \%$ while that for SnPP was $65 \%$ (table 1a).

Both SnPP and SnPPN possess the electron withdrawing phenyl group, which makes the acidic protons more labile, thereby making them better Bronsted acid catalysts. But the surface area of nanomaterial is much higher than the amorphous one. The properties of nanostructure depend strongly on their size and shape. The total number of atoms in a nanoparticle is inversely proportional to the particle size. The surface energy also increases with decreasing particle size. A nanoparticle would prefer to assume a shape which could minimize its surface energy. The activity and selectivity of a catalyst is strongly dependent on the size not only because of the large surface area but also a different electronic structure of the small nanoparticles. When a heterogeneous catalyst is converted into a nanoparticle, the active centres on the
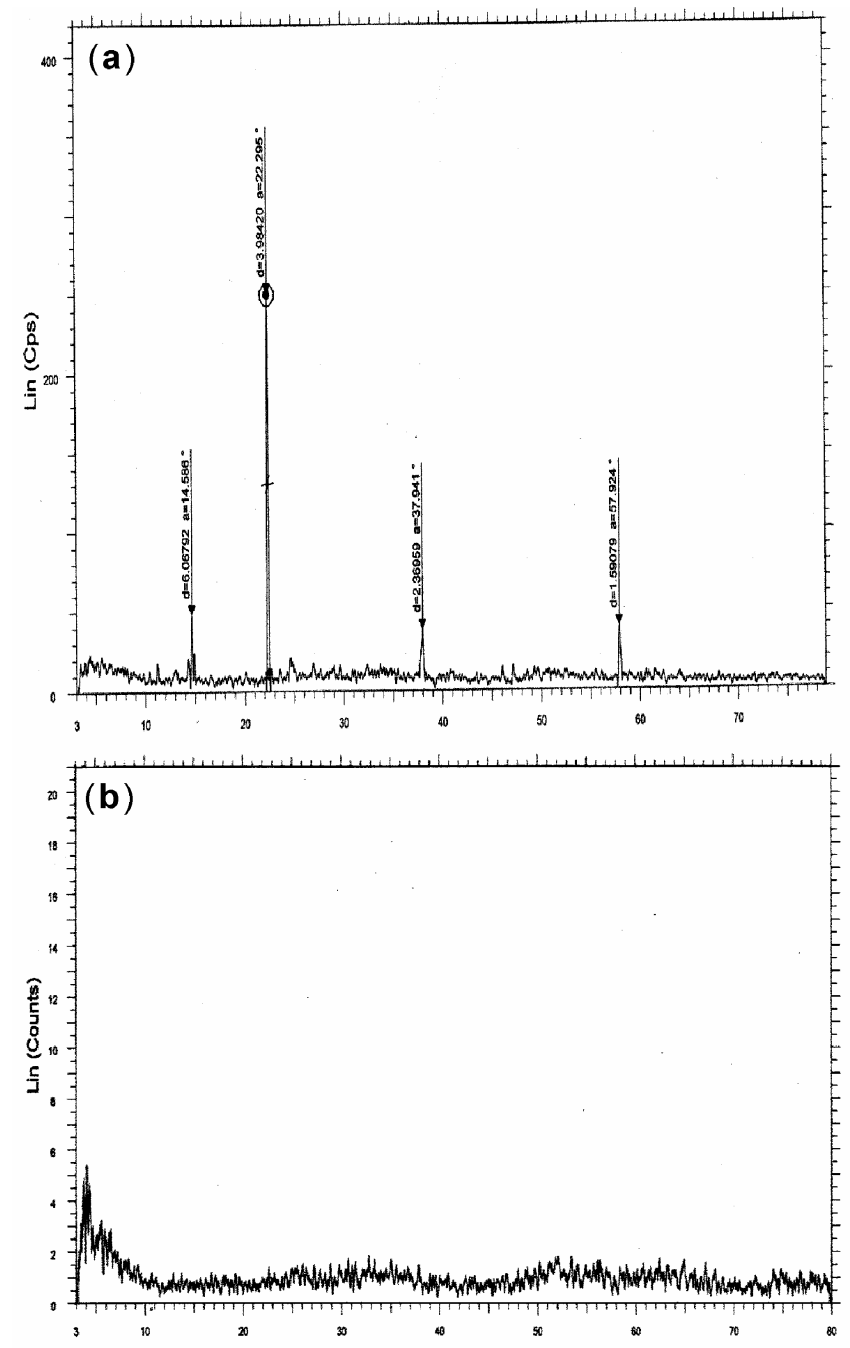

Figure 1. X-ray diffractograms of a. SnPPN and b. SnPP. 

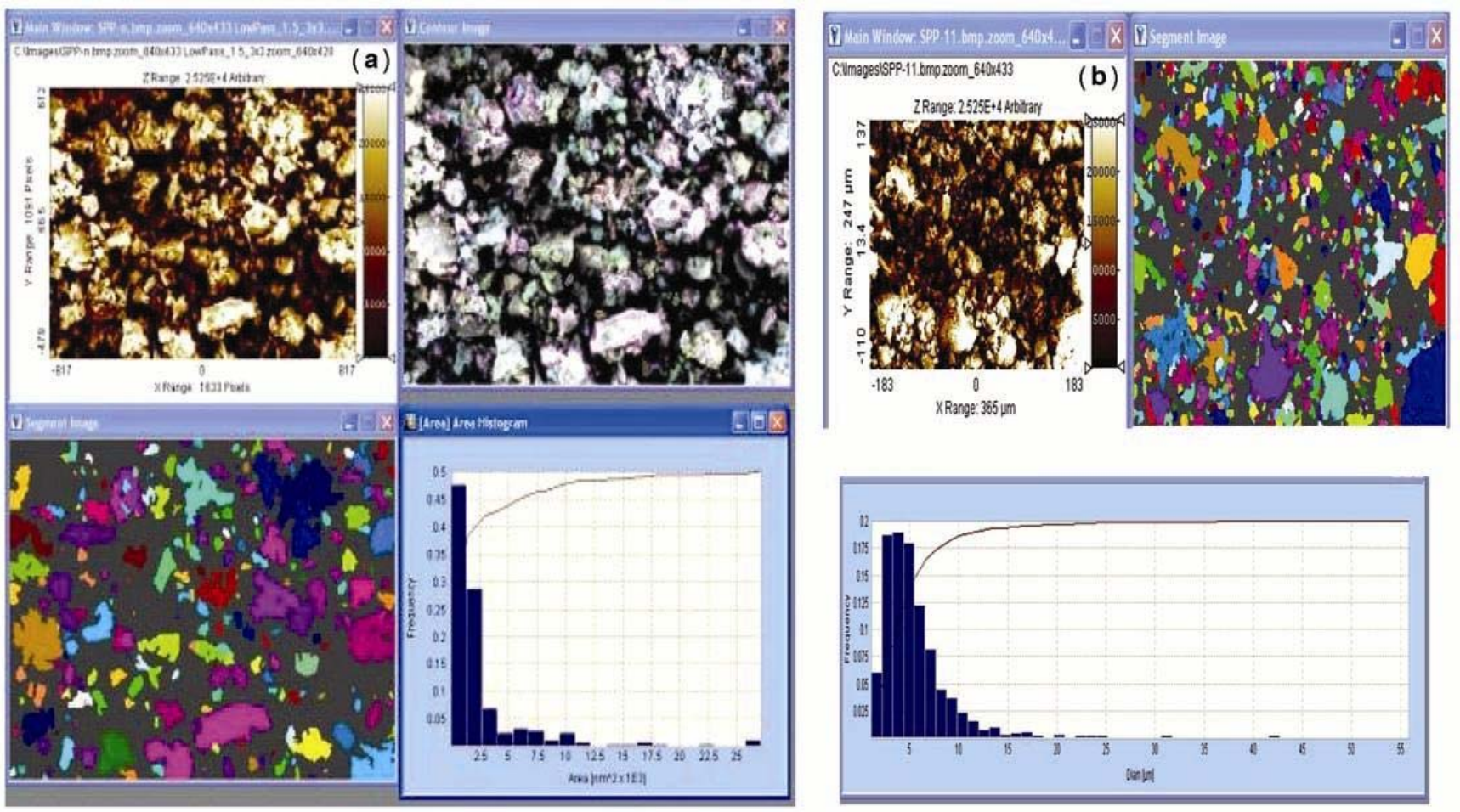

Figure 2. SEM images and particle size distributions of a. SnPPN and b. SnPPA.

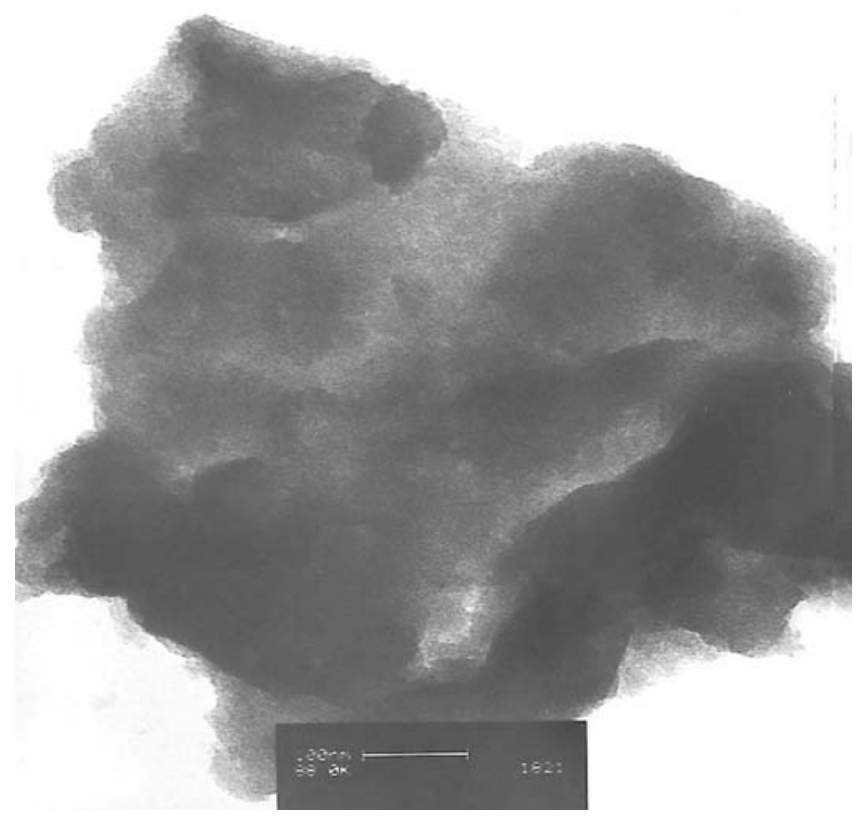

Figure 3. TEM image of SnPPN.

metal surface increases (Anastas and Warner 1998; Anastas et al 2000).

In the case of nanoparticles the edges are catalytically active because the bond between the surface atoms and other atoms in the particle is less strong. This means that

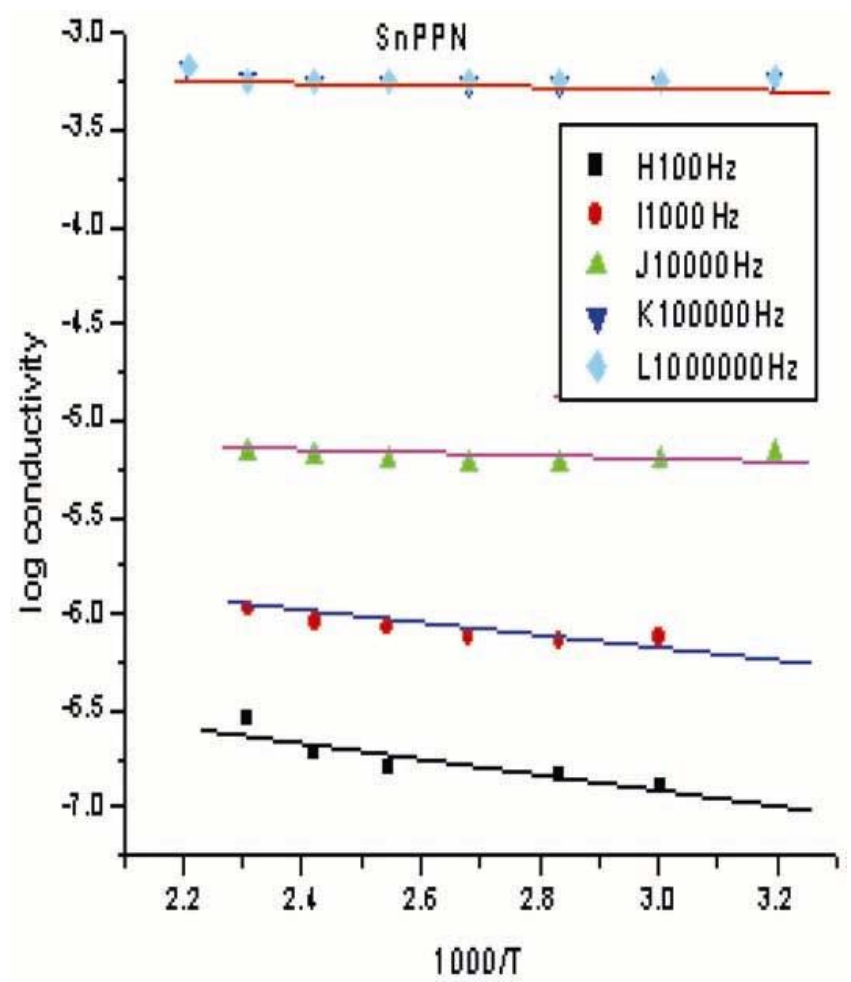

Figure 4. Arrhenius plot for SnPPN.

binding energy is available. The smaller the particles, the greater the number of edges, and the higher the catalytic activity. In the case of SnPP and SnPPN, the surface area 
Table 1a. \% Yield of glycol diacetate using SnPP and SnPPN as catalysts.

\begin{tabular}{lccc}
\hline & & \multicolumn{2}{c}{$\%$ Yield of ester } \\
\cline { 3 - 4 } $\begin{array}{l}\text { Amount of } \\
\text { catalyst }(\mathrm{g})\end{array}$ & $\begin{array}{c}\text { Ratio of acid } \\
\text { to glycol }\end{array}$ & SnPP & SnPPN \\
\hline $0 \cdot 5$ & $1: 1$ & 20 & 30 \\
$1 \cdot 0$ & $1: 1$ & 23 & 36 \\
$2 \cdot 0$ & $1: 1$ & 29 & 45 \\
$0 \cdot 5$ & $2: 1$ & 29 & 46 \\
$1 \cdot 0$ & $2: 1$ & 59 & 64 \\
$2 \cdot 0$ & $2: 1$ & 65 & 75 \\
\hline
\end{tabular}

Table 1b. Variation of conductivity with temperature.

\begin{tabular}{lc}
\hline Temperature $\left({ }^{\circ} \mathrm{C}\right)$ & Specific conductivity $\left(\mathrm{ohm}^{-1} \mathrm{~cm}^{-1}\right)$ \\
\hline 40 & $5.86122 \times 10^{-4}$ \\
60 & $5.66022 \times 10^{-4}$ \\
80 & $5.57028 \times 10^{-4}$ \\
100 & $5 \cdot 56505 \times 10^{-4}$ \\
\hline
\end{tabular}

of latter is higher than the former. The large surface area and the many active sites of SnPPN makes it a very effective catalyst. So this high surface area nanomaterial can be used as an environment friendly solid acid catalyst.

The a.c. conductivity of the material measured at different temperatures is shown in table $1 \mathrm{~b}$. It indicates that conductivity decreases with increase in temperature. This is attributed to the loss of water of hydration as well as the condensation of structural hydroxyl groups with increasing temperature. This suggests the mechanism of transportation to be Grotthuss type (Clearfield 1988) as in the case of SnPP, where the conductivity depends on the ability of water located on the surface to rotate and participate (Schuster and Meyer 2003).

The conductivity of SnPPN at $40^{\circ} \mathrm{C}$ is found to be $5.86122 \times 10^{-4} \mathrm{ohm}^{-1} \mathrm{~cm}^{-1}$, while that of SnPP is only $1.522233 \times 10^{-7} \mathrm{ohm}^{-1} \mathrm{~cm}^{-1}$. As expected the conductivity of SnPPN is higher because of its large surface area. The activation energy $\left(E_{\mathrm{a}}\right)$ for conduction at room temperature is found to be $0.005677 \mathrm{eV}$ for SnPPN while for SnPP its value is $0.0203 \mathrm{eV}$ (figure 2). The order of higher conductivity and lower activation energy matches well in the case of SnPPN. When the activation energy is lower, the proton conducting mechanism needs a lower energy barrier for the Grotthus type diffusion (Patel and Chudasama
2006). Thus the size reduction of the particle increases the catalytic activity as well as conductivity.

\section{Conclusions}

Catalytic activity, recyclability and ease of product separation from the catalyst are considered as the three important criteria in evaluating the performance of catalysts. The high catalytic activities of nano meter-sized metal particles appear promising for industrial and environmental applications. Owing to the size, their electron structure is easily affected by environments such as structure and nature of support, substrates etc. As a consequence of the modified electron band structure, the activity and selectivity of a given catalytic reaction is easily controlled by the bonding between their surface and the reacting molecules (e.g. chemisorption). Nanocomposites like SnPP are promising solid acid catalysts. The nanocomposite materials are also good protonic conductors possessing good conductivity even at $100^{\circ} \mathrm{C}$. Such materials could be used in high temperature applications like hydrogen sensors.

\section{References}

Anastas P T and Warner J C 1998 Green chemistry: theory and practice (New York: Oxford University Press)

Anastas P T, Bartlett L B, Kirchhoff M M and Williamson T C 2000 Catal. Today 5511

Ayyappan and Clearfield Abraham 2005 229th ACS National Meeting (Sandiago, CA, USA) p. 114

Chithra P G and Beena B 2007 Oriental J. Chem. 23339

Clearfield A 1988 Chem. Rev. 88125

Gómez-Alcántara Maria del Mar et al 2007 Dalton Trans. 2394

Guczi L 1989 Proc. 9th Int. congress on catalysis (eds) M J Phillips and M Ternan (Ottawa: Chemical Institute of Canada) p. 114

Haq S, Winkler C, Carew A and Raval R 2004 J. Catal. 1226

Haruta M 1997 Catal. Today 36153

Heemanshu Patel and Chudasama Uma 2006 Bull. Mater. Sci. 29665

Honma I, Nomura S and Nakajima H 2001 J. Mem. Sci. 18583

Huang H H, Wilkes G L and Carlson J G 1989 Polymer 30 2001

Ribeiro F H, von Wittenau A E S, Bartholomew C H and Somorjai G A 1997 Catal. Rev. Sci. Eng. 3949

Sakurai H and Haruta M 1996 Catal. Today 29361

Schuster M F H and Meyer W H 2003 Rev. Mater. Res. 33233

Turkevich J and Kim G 1970 Science 169873 